\title{
Smart Technology and Industry Trends in Fourth Industrial Revolution
}

\author{
Seong-Hoon Lee, Hyun-Soo Jin
}

\begin{abstract}
In this paper, we have been studying the technology trends closely approaching us and the smart machine, which is a representative example of the forth industrial revolution. The key content in technology trends is artificial intelligence. Artificial intelligence technology is being used as a core technology of the 4th industrial revolution. In autonomous vehicles, which are representative industries, artificial intelligence-based unmanned vehicles are emerging, and on the other side, and various voice recognition based products are emerging. In this paper, we have studied the latest technical factors of autonomous automobile and speech recognition based industry, which is a representative industry of the 4th industrial revolution.
\end{abstract}

Keywords : Smart machine, Machine intelligence, IoT, Fourth revolution.

\section{INTRODUCTION}

$\mathrm{W}$ ith the advent of Google AlphaGo, there is a growing interest in intelligence in our society. Industry and research institutes are pursuing a more human-oriented direction using intelligence information, and researches such as intelligence-related research and departmental organization are being made in academia. In addition, various smart machines are emerging based on smart technologies to build a smart society [1]. Such a smart machine is a word that combines the word 'smart' and the word 'machine', and has been widely used in our society as a smart machine or a smart machine with intelligence.

When analyzing the necessary elements of a machine to be smart, there are basically computing technology, storage device, communication technology and sensing technology. In addition, various technologies necessary for the implementation of functions required for each product may be included. Machine intelligence enables intelligent judgment and autonomous actions to be performed by recognizing the context and analyzing the recognized information by utilizing the sensing function generated by combining existing products with IT technology [2], [3]. Therefore, smart machines act autonomously, equipped with intelligence and self-learning functions, self-judging and

Revised Manuscript Received on July 22, 2019

* Correspondence Author

Seong-Hoon Lee*, Division of ICT, Baekseok University, Cheonan, Korea.Email: shlee@bu.ac.kr

Hyun-Soo Jin, Division of ICT, Baekseok University, Cheonan, Korea. Email: jhs1020@bu.ac.kr adapting according to the situation, and can perform tasks that they thought could only be done so far.

The types of smart machines can be roughly divided into smart machines represented by movements and machines without such machines. Examples of electronic machines include autonomous vehicles and unmanned helicopters. An example of the latter smart machine is Apple's Siri, which acts as a visual assertion of the day's weather and schedule when asked by voice, IBM's Watson as an advisor to advise on appropriate diagnosis with clinical opinion or test data.

In this paper, we analyze the changes of the technology and applied techniques in forth industrial revolution, and also describe the trends of related industries.

\section{TECHNOLOGICAL CHANGES}

According to data from Gartner, which announces technology trends that can be a global issue each year, there is no big change in core technology from 2017 to 2018 [4], [5]. Therefore, current technology trends will be a key category for future global market. The changes in technology trends in 2017 and 2018 can be seen in the table- I and in Fig. 1 below. Key keywords commonly seen in the two years are Intelligent, Digital, and Mesh.

Table- I : Change of technologies on 2017 2018

\begin{tabular}{|c|c|c|c|}
\hline & $\begin{array}{c}\text { 2017 Strategic } \\
\text { technology }\end{array}$ & $\begin{array}{c}\text { 2018 Strategic } \\
\text { Technology }\end{array}$ & remarks \\
\hline \multirow{4}{*}{ Intelligence } & $\begin{array}{c}\text { Applied AI \& } \\
\text { Advanced } \\
\text { Machine Learning }\end{array}$ & AI Foundation & $\begin{array}{c}\text { AI } \\
\text { Reinforcement }\end{array}$ \\
\cline { 2 - 4 } & Intelligent Apps & $\begin{array}{c}\text { Intelligent Apps } \\
\text { \& Analytics }\end{array}$ & \\
\cline { 2 - 4 } & Intelligent Things & $\begin{array}{c}\text { Intelligent } \\
\text { Things }\end{array}$ & $\begin{array}{c}\text { Intelligent } \\
\text { Device }\end{array}$ \\
\hline \multirow{4}{*}{ Digital } & AR/VR & Digital Twins & \\
\cline { 2 - 4 } & Digital Twins & $\begin{array}{c}\text { Cloud to the } \\
\text { Edge }\end{array}$ & $\begin{array}{c}\text { Edge } \\
\text { Computing }\end{array}$ \\
\cline { 2 - 4 } & $\begin{array}{c}\text { Block-chain\&Dist } \\
\text { ributed Ledgers }\end{array}$ & $\begin{array}{c}\text { Conversational } \\
\text { Platforms }\end{array}$ & \\
\cline { 2 - 5 } & $\begin{array}{c}\text { Conversational } \\
\text { System }\end{array}$ & $\begin{array}{c}\text { Immersive } \\
\text { Experience }\end{array}$ & VR, MR \\
\hline \multirow{2}{*}{ Mesh } & $\begin{array}{c}\text { Mesh App \& } \\
\text { Service } \\
\text { Architecture }\end{array}$ & BlockChain & \\
\hline
\end{tabular}




\begin{tabular}{|c|c|c|c|}
\hline & $\begin{array}{c}\text { Digital } \\
\text { Technology } \\
\text { Platforms }\end{array}$ & $\begin{array}{c}\text { Event-driven } \\
\text { Model }\end{array}$ & $\begin{array}{c}\text { Business } \\
\text { Model }\end{array}$ \\
\cline { 2 - 4 } & $\begin{array}{c}\text { Adaptive Security } \\
\text { Architecture }\end{array}$ & $\begin{array}{c}\text { Continuous } \\
\text { adaptive risk and } \\
\text { Trust }\end{array}$ & Security \\
\hline
\end{tabular}

\section{TOP 10 STRATEGIC TECHNOLOGY TRENDS}

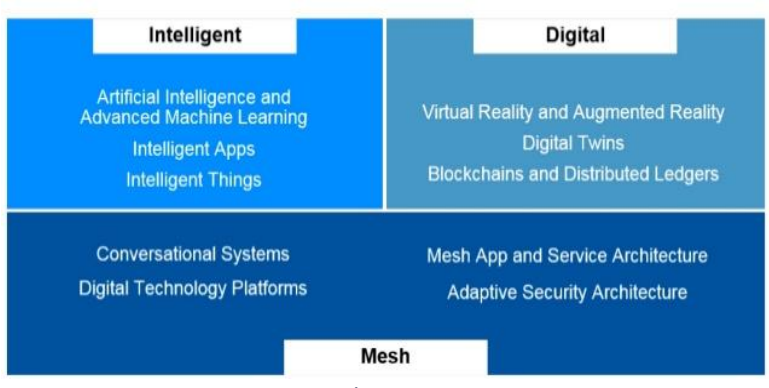

1) 2017

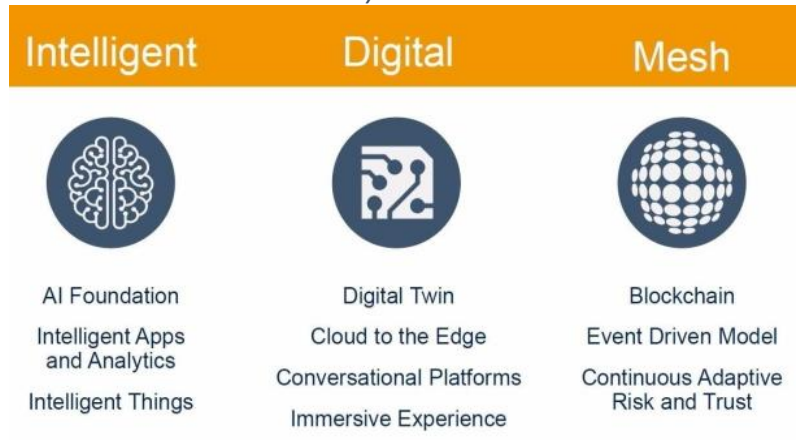

2) 2018

Fig. 1. 2017 2018 Strategic technologies(source: Gartner)

The following is a brief description of Gartner's 2018 Strategic Ten technologies. First, the major trends in strategic technology trends fall into three categories: Intelligent, Digital, and Mesh. According to these categories, ten detailed technologies for each area were selected.

First, intelligence means that artificial intelligence can be applied to all new technologies as well as existing technologies. Second, digital represents the convergence of the virtual world with the real world, which creates immersive experiences. In the final direction, Mesh represents technologies related to strengthening connections between people and business (devices, content, and services) to create digital business results. The three major categories of technologies that represent strategic technology direction are as follows. First, the technology trend related to Intelligent included three technologies.

- AI Foundation: The ability of artificial intelligence in various areas will lead to successful digital initiatives. Obviously, interest in artificial intelligence is increasing. Artificial intelligence technology is rapidly evolving, so in the industrial field, we have invested heavily in these technologies, processes, tools (data preparation, integration, algorithms, training methodologies, model generation, etc.) Should be.

- Intelligent Apps and Analytics: Over the next few years, all app and application services will include a certain level of artificial intelligence. Some apps will be intelligent apps that include artificial intelligence and machine learning. Artificial intelligence has become an important battleground for a wide range of software (SW) and services markets, including enterprise resource planning (ERP).

- Intelligent Things: Intelligent objects use artificial intelligence and machine learning to show advanced functions through artificial intelligence and mean things that people and the environment interact naturally with each other. Artificial intelligence is driving the development of a variety of intelligent objects such as autonomous vehicles, robots, drones, and healthcare, and it is developing the abundance of things such as Internet (IoT), connected consumer, and industrial system.

The second major technology trend is the digital phenomenon, and the related technologies are as follows.

- Digital Twin: A digital twin is a digital representation of a real system. Digital twin will be useful in the next three to five years for IoT projects. In the context of IoT, the digital twin will connect with real objects to provide information to the other party, respond to change, improve operations and add value. Systematically designed digital tweens not only dramatically improve corporate decision making, but can also be linked to physical models that exist in the real world, providing insight into how the object or system is operating, how to use the product, and how to improve it.

- Cloud to the Edge: Edge computing refers to a computing topology in which information processing and content collection and delivery are closely aligned with information sources. Connectivity and latency issues, bandwidth constraints, and various functionalities built into the edge are preferred to the distributed model, and enterprises must use edge computing in infrastructure architectures for those with IoT factors.

- Conversational Platforms: Interactive platforms will lead to the next wave of paradigm shifts between the digital world and human interaction. After receiving a question or command from the user, the platform performs the task of performing the function, presenting the content, and requesting additional input. Over the next few years, the interactive interface will become a major design goal for user interaction and will be delivered on dedicated hardware, core operating system (OS) functionality, applications, and platforms.

- Immersive Experience: Augmented Reality (AR), Virtual Reality (VR), and Mixed Reality (MR) are changing the way people recognize and interact with the digital world. Combined with the interactive platform, there is a radical change from the user experience to an invisible immersive 
experience. High interest in this has led to the emergence of new VR applications (video games, 360-degree rotating video, etc.) that have superior entertainment capabilities, but that are not real business value. Over the next five years, users will focus on a complex reality that emerges as a vibrant experience that interacts with the digital and physical world while maintaining the physical environment.

A third typical technology trend is mesh, and related technologies are as follows.

- Block-chain: The Block-chain is evolving from a digital currency infrastructure to a digital innovation platform with a decentralized account that excludes business friction, regardless of individual applications or participants. It provides the foundation for an innovative digital business for start-ups and start-ups that can move away from the current centralized transaction and record management mechanisms. Though the financial industry is mainly focused on block chains, it is a technology that can be used for government, medical, content distribution, and supply chain.

- Event-Driven: Digital business relies on its ability to leverage new digital businesses. Business events can be tracked and analyzed quickly using event brokers, IoT, cloud computing, and artificial intelligence, all of which can be digitally reflected in notable conditions such as product purchase orders, aircraft takeoffs, and changes.

Continuous and Adaptable Risk - Trust Assessment (CARTA) Approach: Digital business creates a complex and evolving security environment. In order to securely drive digital business initiatives in a world where more sophisticated targeted attacks are possible, security and risk management personnel need to be able to adapt to the risk and confidence assessment (CARTA) that can adapt continuously to make real-time threats and reliabilityApproach. Security infrastructures must be adaptable everywhere for opportunity capture and risk management through providing security that moves at the same speed as digital business.

\section{INDUSTRY TRENDS}

\section{A. Autonomous Vehicle}

Just a few years ago, the criteria for choosing a car were robustness, safety, size and design. In recent years, the trend of high oil prices has continued and eco-friendly vehicles have been attracting attention, and average mileage per liter of fuel, that is, fuel efficiency has become a hot topic of automobile technology. The younger generation, who are aiming at rational consumption, should choose a car that is more fuel efficient considering the maintenance cost rather than the bigger displacement. The fact that the price cuts of imported cars and advanced fuel economy techniques appeal to the younger generation also encourages this phenomenon.
Smart car system technology, which automatically drives, stops and parks, is also becoming a reality as competition for fuel economy improvement technologies and eco-friendly technologies such as hybrid cars and electric cars are expected to become more intense [6], [7].

Recent conferences show that the convergence between automobiles and electronic components is becoming an irresistible trend, with Volkswagen's autonomous vehicles, Mercedes-Benz's drones, and Toyota's artificial intelligence-coupled concept cars. The final destination of automobile - based technology convergence can be said to be autonomous driving further from this movement of electricalization.

An autonomous drive car is a car that can recognize the environment, judge the route and the danger, and operate safely, even if the driver does not control the main device [8].

As the automotive market combines various software and content, large IT companies such as Apple and Google are expanding into the automotive field. Apple and Google are seeing the connected car market as a future growth engine and are running an infotainment platform based on its Smartphone operating system.

Apple's CarPlay and Google's Android Auto, along with the display touch method, utilize its advanced voice recognition features to connect with the Smartphone, Continuous updates are available. In addition, it has the potential to innovate the automotive platform in the future by offering a variety of services and content through the App Store.

Ultimately, if people do not drive and enter only the goal point, research that can go on their own is also active. Google unveiled an unmanned car with no handle, no accelerator pedal, no brakes, no start, no stop button, and then moved to the destination after talking to the destination. A driver in a car is not actually driving, so it is better to call it an occupant. After you get on the car, just tell your destination and the car will take you to your destination.

Google's unmanned automotive testing is aimed at enabling roads to run on the road in a few years with continuous testing and improvement. Of course, there should be institutional conditions for unmanned vehicles to run on public roads. This institutional aspect has been further improved.

Meanwhile, automobile companies in developed countries such as Mercedes Benz, Audi and Volvo have already commercialized car distance, pedestrian recognition, speed control, and automatic parking technology through long-term research and development. It is not a completely unmanned automobile, but it is a step to go to an unmanned car in that it is partly self-judging and moving. Automotive radar, cameras, sensors, and software are key technologies that enable these functions.

Among these, the technology that can prevent a car accident is an autonomous emergency braking system (AEB). The technology, called a traffic accident response system, uses radar, laser, and video to calculate that a traffic accident is imminent. If the 
driver's preset distance is not maintained, the software will automatically operate the brake. Volvo Cars demonstrated the 'City Safety' function, the first of its kind in the world. It is essential not only to maintain the distance between cars but also to reduce the speed of the vehicle itself when a pedestrian appears.

The state-of-the-art facilities that help ensure safe driving are coming into the car. Here, a technology for analyzing a driver's bio-signal is utilized. Technology has also been shown to prevent drowsiness, a major cause of highway deaths. The drowsy driving alarm recognizes the driver's blinking speed and focus and alerts the drowsy driving.

It also analyzes the blood alcohol concentration through driver's eye movement, handle operation, and driver breathing, and measures alcohol drunk driving to determine whether to drive or not to reduce speed or start at all. The system developed by Toyota of Japan analyzes the blood alcohol concentration by analyzing the sweat component of the driver's hand holding the steering wheel before starting the operation. A system that detects the driver's eye movements and automatically stops when the focus is overshadowed by drinking is also applied to some cars.

The 'smart' car that enables safe operation while moving on its own has become a reality in front of us because of advances in advanced science and technology and advances in computer science to deal with so-called 'big data'. It is approaching the time when it can get away from the traffic jam and can see the book and work on the car.

The development of connected cars is predicting automobiles as connected devices. At present, vehicle control and monitoring services are installed in vehicles, and media content streaming and various application services are mainly used in connection with Smartphone. Ultimately, it is expected that all the connectivity, platforms and solutions will be built into the vehicle and the car itself will evolve into a 'Connected Device'.

\section{B. Voice Recognition based Services}

Voice recognition technology refers to a technique in which a computer converts an acoustic voice signal obtained through a sound sensor such as a microphone into a word or a sentence [9]. Fig. 2 below shows a typical voice recognition process.

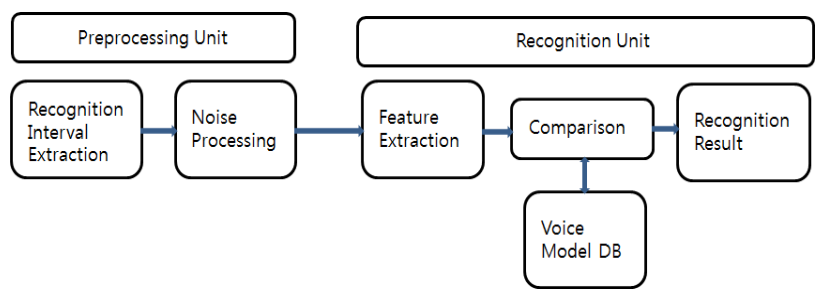

Fig. 2. Voice recognition process

Generally, voice recognition technology extracts acoustic signals and then removes noise. Then, voice recognition is performed by extracting features of a speech signal and comparing with the speech model database (DB). Voice recognition technology is also a combination of sensing and data analysis technology, but it is known that it is easier and more accurate to grasp human intentions because the data to be measured and analyzed is one voice data.

Various voice recognition services based on voice recognition technology began to be introduced in the late 2000s. A typical example is Siri, Apple's voice-based personal assistant service launched in 2011. Siri is a personal assistant service that provides various services such as calendar search, dialing, memo, music playback as well as mobile search based on voice commands of iPhone users. Since the launch of Apple's Siri, Google has launched a personal assistant service based on voice recognition, such as Google Now and Microsoft, and Cortana, and Japan has also launched a foreign language interpretation service. Samsung Electronics in Korea and LG Electronics have developed a voice recognition service with similar functions. These voice recognition services are developed to serve as voice interfaces for various application services rather than services.

A virtual assistant is a software agent that processes user-requested tasks and provides user-specific services like a personal assistant [10], [11]. Based on the AI engine and voice recognition, it collects and provides customized information to the user and performs various tasks such as schedule management, e-mail transmission, and restaurant reservation according to the user's voice command. In addition, it is expected that the application range will be further expanded by being mounted on various smart home appliances and vehicles.

According to Eileen Brown's article, a professional consultant who cited the results of a recent survey by Stone Temple, marketing and search engine optimization (SEO) specialist, Stone Temple compares the intelligence of Google Home and Amazon Echo Research was conducted. And subsequent studies contrasted the smartness of Google's assistant (home), Apple Shiri, MS Kotana and Amazon Alexa (echo). Both studies did not cover the recently released Apple Smart speaker 'HomePod' [12].

The study concluded by calculating the appropriateness of the answer, which people would routinely ask for in a personal assistant product, or a questionnaire that was based on factual knowledge that was searched on Google.

Stone Temple measured the question response rate of the four AI virtual assistants and the accuracy of the questions answered. The Google assistant on the Google Home accounted for a response rate of $68.1 \%$ and accuracy of $90.6 \%$. MS Kotana had a response rate of $56.5 \%$ and accuracy of $81.9 \%$. Apple's Shiri response rate was $21.7 \%$ and accuracy was $62.2 \%$. Alexa on Amazon Echo had a response rate of $20.7 \%$ and accuracy of $87.0 \%$.

According to Eileen Brown's experiments, Google Home and Google Assistant are the winners. MS Kotana was a little less than that, but in recent years it has been rated as having dramatically evolved into a rich answer.

However, it is not appropriate to use this research as an absolute index for judging the level of products or 
technology in the AI virtual assistant market. It is difficult to rule out the possibility that the experiment itself was designed to favor Google.

And this study suggests that Apple may be subject to any change in technology as a result of the work done prior to the World Development Conference (WWDC) 2017, which introduced a clearly improved version of the iOS11 version.

\section{CONCLUSION}

At present, our society has made a lot of progress through the third industrial revolution. With the economic growth through industrialization, the size and quality of life can be matured. However, the term "tertiary industrial revolution" is now turning into a new paradigm called the Fourth Industrial Revolution.

At this turning point, we can conclude that current intelligent technologies can be applied to new technologies as well as existing technologies. Secondly, digital is a virtual world that creates immersive experiences. It represents fusion with the real world. In the final direction, Mesh represents technologies related to strengthening connections between people and business (devices, content, and services) to create digital business results.

The fourth industry is basically converged with ICT technology, which essentially includes intelligence information, and a variety of smart machines based on Internet technology will be a good example. In this study, we examined the contents related to autonomous driving, which is a representative example of two types of smart machines, and the technology and industry trends required for voice recognition based services.

\section{REFERENCES}

1. D. H. Kim, Smart Technology, LG CNS, 2010.

2. H. S. Lee, Possibility of Future Industry, Smart Technology, LG CNS, 2010.

3. H. B. Jang, Current Status and Prospect of Smart Technology, LG CNS, 2011.

4. Gartner, The Top Ten Strategic technology Trends in 2017, 2016 Available https://www.gartner.com/doc/3471559/top--strategic-technology-trends.

5. Gartner, The Top Ten Strategic technology Trends in 2018, 2017 Available from: https://www.gartner.com/smarterwithgartner/gartner-top-10-strategic-tech nology-trends-for-2018

6. M. Andrew, Automotive Industry Trends: IoT Connected Smart Cars \& Vehicles, UK Business Insider, 2017.

7. Auto Connected Car News, Definition of Connected Car-What is the Connected Car? Defined, AUTO Connected Car, 2014. Available from: http://www.autoconnectedcar.cpm/definition-of-connected-car-what-is-the -connected-car-defined/

8. S. E. Baeck, "The Outlook of Software at the era of Autonomous Driving", Entrue Journal of Information Technology, 16(1), 7-33, 2017.

9. V. Zwas, Speech Recognition Technology, Encyclopedia Britannica Online, Britannica.com, 2016. Available from: https://www.britannica.com/technollogy/speech-recognition

10. G. Chaim, Apple announces HomePod Speaker to take on Sonos, The Verge, 2017.

11. M. B. Hoy, Alexa, Siri, Cortana, and More: An Introduction to Voice Assistants, Medical Reference Services Quarterly, 37(1), 81-88, 2017. https://doi.org/10.1080/02763869.2018.1404391

12. B. Eileen, Google Home vs Amazon Alexa: Which one is Smarter?, 2017. Available https://www.zdnet.com/article/google-home-versus-amazon-alexa-whichone-is-smarter/.

\section{AUTHORS PROFILE}

Seong-Hoon Lee received his M.S. and Ph.D. degrees in Computer Science and Engineering from Korea University, Korea, in 1995, and 1998, respectively. He has been a professor in Division of ICT, Baekseok University. His research interests include distributed computing, web service, mobile computing.

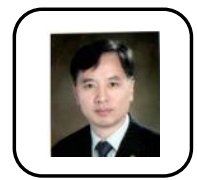

Hyun-Soo Jin received his M.S. and Ph.D. degrees in Electric Engineering from University of Seoul, Korea, in 1992, and 2000. He has been a professor in Division of ICT Baekseok University. His research interests are information communication and $\mathrm{AI}$ 